\title{
3D modelling of leaves from color and ToF data for robotized plant measuring
}

\author{
G. Alenyà, B. Dellen and C. Torras
}

\begin{abstract}
Supervision of long-lasting extensive botanic experiments is a promising robotic application that some recent technological advances have made feasible. Plant modelling for this application has strong demands, particularly in what concerns 3D information gathering and speed. This paper shows that Time-of-Flight (ToF) cameras achieve a good compromise between both demands, providing a suitable complement to color vision. A new method is proposed to segment plant images into their composite surface patches by combining hierarchical color segmentation with quadratic surface fitting using ToF depth data. Experimentation shows that the interpolated depth maps derived from the obtained surfaces fit well the original scenes. Moreover, candidate leaves to be approached by a measuring instrument are ranked, and then robot-mounted cameras move closer to them to validate their suitability to being sampled. Some ambiguities arising from leaves overlap or occlusions are cleared up in this way. The work is a proof-of-concept that dense color data combined with sparse depth as provided by a ToF camera yields a good enough 3D approximation for automated plant measuring at the high throughput imposed by the application.
\end{abstract}

\section{INTRODUCTION}

Recent advances in depth sensors [1], deformable object modelling [2], and autonomous mobile manipulation [3] have considerably widened the scope of robot application. One area that is nowadays gaining attention since it could benefit from all these advances is the monitoring and maintenance of large botanic experimentation fields, e.g., for plant phenotyping. The goal is to determine the best treatments (watering, nutrients, sunlight) to optimize predefined aspects (plant growth, seedling, flowers) and, towards this aim, experiments entailing many repetitive actions need to be conducted [4]. Measurements and samples from leaves must be regularly taken and some pruning needs to be performed. These are tasks for which robots would be very handy, difficulties arising from the complex structure and deformable nature of plants, which do not only change appearance through growing, but whose leaves move also on a daily cycle.

As regards to sensing, color vision has been used to obtain some relevant plant features, but when it comes to extracting structural/geometric information for modelling and manipulation purposes, the concourse of a user is required to provide hints on segmentation from multiple views [5]. If a

This research is partially funded by the EU GARNICS project FP7247947 , by the Spanish Ministry of Science and Innovation under projects DPI2008-06022 and MIPRCV Consolider Ingenio CSD2007-00018, and the Catalan Research Commission. G. Alenyà was supported by the CSIC under a Jae-Doc Fellowship. B. Dellen acknowledges support from the Spanish Ministry for Science and Innovation via a Ramon y Cajal fellowship.

Authors are with Institut de Robòtica i Informàtica Industrial, CSIC-UPC, Llorens i Artigas 4-6, 08028 Barcelona, Spain; $\{$ galenya, bdellen, torras\}@iri.upc.edu

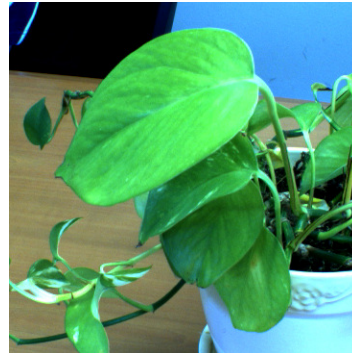

(A) Color image

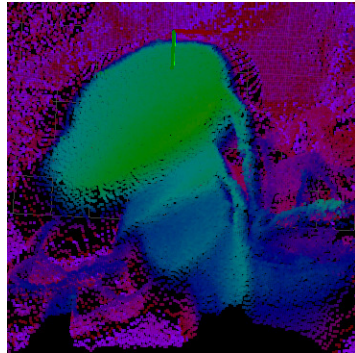

(B) Point cloud
Fig. 1. Images of a plant as acquired by a color camera and a ToF camera. Note the slight difference in viewpoint due to the unavoidable physical separation of the two cameras. (A) Color image: some leaves appear superimposed, which makes segmentation and area measurement difficult (B) ToF image: 3D point cloud colored with the real depth values.

fully automated process is sought, depth information needs to be extracted through stereo [6], structured light [7] or a laser scanner. These techniques have proven adequate for offline modelling, but are too slow to be used in online robot interaction with plants. Recently, Time-of-Flight (ToF) cameras have been proposed as a good alternative [8], [9], since they provide low-resolution depth images at 25 frames-per-second. This permits quickly acquiring and fusing images from different viewpoints [10], which is very useful since one-shot plant data are often partial or ambiguous. Some works have exploited the best of both technologies by combining ToF data with high-resolution color images to deliver dense depth maps [11], [12].

Concerning robot action, planning and learning algorithms for the manipulation of deformable objects [13] are deemed to play an important role in this context. Planning needs to encompass the motion of the camera as well, since plants are prone to occlusions and merging of close leaves, so that selecting the best next viewpoint may be crucial to determine and access a suitable probing point.

Along this line, the European project GARNICS

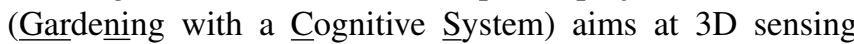
of plant growth and building perceptual representations for learning the links to actions of a robot gardener. This paper focuses on the sensing-for-action methods developed to segment leaves, fit quadratic surfaces to them, determine best candidates for probing, move the cameras to get a closer view, determine a suitable sampling/measuring point on the chosen leaf, and finally reach this point with a probing tool. Color-based segmentation is complemented with depth data supplied by a ToF camera (Fig. 1) to delimit and fit surface patches to the leaves. The color and ToF cameras, as well as 


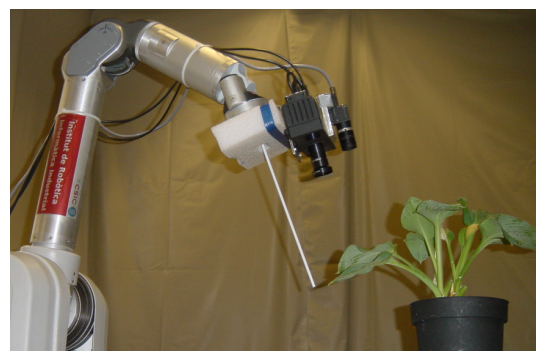

Fig. 2. WAM arm used in the experiments holding the color and ToF cameras, as well as a fake measurement instrument in the form of a stick.

the probing tool, are all mounted on the robot end-effector, so that an egocentric coordinate frame is used for all motions.

The paper is structured as follows. In Section II, an overview of the system is provided. Next, in Section III, we explain how images obtained with the depth and the color camera are fused. In Section IV, the depth-aided color segmentation algorithm is described, followed by Section V, where the plant monitoring procedure is explained. Experimental results are presented in Section VI and discussed in Section VII.

\section{SYSTEM OVERVIEW}

The experimental setup includes a PMD CamCube Timeof-Flight camera and a PointGrey Flea camera rigidly attached to the last link of a Barrett WAM arm (Fig. 2). As can be observed, the cameras are displaced from the robot end-effector position to leave room for the future placement of measuring devices, which are here replaced by a $30 \mathrm{~cm}$ long stick.

The aim of the present work is to develop a completely automated procedure to segment and model some leaves of a plant using a general view and possibly other closeup views obtained by moving the camera system with the robot arm. For the general view, we assume that the plant is located in a known position, so that the first image is always taken from the same point of view. This is a reasonable assumption since, in large phenotyping facilities, plants are usually located in multiple-position trays or they move in a conveyor belt. Close-up views are taken to verify the accessibility of the best leaf candidates, by removing ambiguities due to merged leaves or occlusions.

The process consists of three stages. First, color and depth images are acquired and combined to obtain a colored point cloud. The method to determine the required calibration matrices $\mathbf{K}_{\mathbf{r g b}}$ and $\mathbf{F}$ is explained in Section III.

Second, the different leaves are segmented from the point cloud, and a plane or a quadratic surface is fitted to each of them. The surface model provides the position and orientation of each leaf. This first segmentation may contain some errors, e.g., several superimposed leaves may fall in the same region, and regions including few points may lead to a relatively large fitting error.

Third, using the position and orientation of the best leaf candidate, the robot moves the camera system closer to it to obtain a more detailed view, which is used to obtain a better model and eventually separate different leaves. The method to determine the required transformation matrices $\mathbf{H}_{11}$ and $\mathbf{H}_{12}$ is explained in Section V-B.

In the future we will consider also the images perceived along the robot motion from the initial view to the detailed view. In this framework, the use of a time-of-flight camera will be advantageous.

\section{IMAGE FUSION}

The cameras need to be calibrated in order to fuse depth and color information. Taking advantage of the fact that the ToF camera provides registered depth and grey-level images, we apply a standard stereo calibration algorithm making use of a small calibration pattern at close distances. The calibration error is lower for the RGB camera than for the ToF camera due to the reduced resolution of the latter, so to perform the internal parameter calibration more sample images are captured with the ToF camera to try to compensate for this to some extent. With this procedure, the required matrix $\mathbf{K}_{\mathbf{r g b}}$ of intrinsic parameters is determined, and also the extrinsic matrix $\mathbf{F}$ that in our case provides the position of the ToF camera with respect to the color camera.

The fusion of the depth with the color data is performed by transforming the $3 \mathrm{D}$ point coordinates to the RGB camera reference frame, and then projecting these points to the camera image plane. Within this framework, color points not having a 3D counterpart are discarded. If necessary, denser point clouds can be obtained through linear interpolation between consecutive 3D points or using a more elaborate method based on 3D meshes [14].

However, as both cameras have slightly different viewpoints (Fig. 1), some points are seen by one camera but not by the other, and consequently it is impossible to find their correspondence. These occlusions appear mainly for closer objects, precisely our scenario.

In our method they are detected and removed using a Z_buffer approach. First, the point cloud is transformed to the RGB camera reference frame using the extrinsic transformation matrix F. Ideally, this leads to 3D points projecting on the corresponding pixel in the color image. In the case of occlusion, only the point that is closer to the camera is stored in the Z_buffer. However, as the ToF camera has a lower resolution $(204 \times 204)$ than the color camera $(640 \times 480)$, two different $3 \mathrm{D}$ points (namely, the foreground and the occluded background points) may not project exactly onto the same color point, so no one is removed. This leads to a mosaic of foreground-background pixels in the regions where occlusions occur. We have taken into account a neighborhood region to build the Z_buffer, so that the depth of neighbours determine whether occlusions are to be considered (Fig. 3).

For very close views, we further removed all 3D points close to the background (using background subtraction) to enforce the suppression of any wrong colored point.

\section{DEPTH-AIDED COLOR SEGMENTATION}

We describe an algorithm for the segmentation of color images into surface patches using sparse depth data. Images 


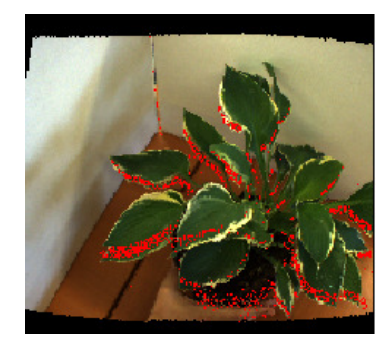

Fig. 3. Result of assigning to each 3D point its corresponding color. Occlusions, i.e., depth points that are not viewed by the color camera, are marked as red points.

are segmented in the color space, but the segmentation procedure is aided by the depth data. Since the segmentation is performed in the dense color space, even sparse or noisy depth information can be used. This kind of data often poses problems to segmentation algorithms operating in the depth data space directly. The core idea of our method is based on the notion that surface boundaries are in most cases represented by an edge in the color image. Since we are dealing with sparse depth data, it is further desirable to have as large segments as possible - otherwise model fitting becomes impracticable due to lack of data inside segments. We thus segment the color image with different resolutions (see Section IV-A). Quadratic surface models are fitted to each segment (Section IV-B), and we select those segments from the hierarchy which minimize the total fitting error, while taking into account the hierarchy level, i.e., segments obtained at lower resolutions are given preference to segments at higher ones (Section IV-C). The resulting new segmentation is then further improved by applying an additional region merging step through which segments having highly different color values can be merged if they describe the same surface (see Section IV-D). Then, unclustered points are assigned to the closest surface, using both depth and color information (see Section IV-E).

\section{A. Hierarchical color segmentation}

The color image is segmented using the method of superparamagnetic clustering of data employed in [15] which allows a segmentation hierarchy to be generated by segmenting the image with different resolutions. For this purpose, we varied the interaction strength by multiplying the mean distance $\bar{\triangle}$ by a factor between 1.4 and 0.6 .

\section{B. Model fitting and selection}

For each color segment $s_{i}$ and model type (see Section IVF) we perform a minimization of the mean square distance

$$
E_{i, \text { model }}=1 / N \sum_{j}\left(z_{j}-z_{j, m}\right)^{2}
$$

of measured depth points $z_{j, m}$ from the estimated model depth $z_{j}=f_{i \text {,model }}\left(x_{j}, y_{j}\right)$, where $f_{i \text {,model }}$ is the data-model function and $N$ is the number of measured depth points in the area of segment $s_{i}$. The optimization is performed with a Nelder-Mead simplex search algorithm.
The mean square errors for two different model types, i.e. $E_{i \text {,plane }}$ and $E_{i \text {,curved }}$, are computed. We select the planar model if $E_{i \text {,plane }}<E_{i \text {,curved }}+\tau_{1}$, the curved model otherwise. The larger parameter $\tau_{1}$, the more preference is given to planar surfaces than to curved surfaces.

\section{Color-segment selection procedure}

We define the following selection procedure considering first only two levels $u$ and $u+1$, where a higher level denotes a higher resolution. Let $s_{i}^{u}$ be a segment at level $u$ of the hierarchy having a fitting error $E_{i}^{u}$ (see Section IV-B). At the level $u+1$ of the hierarchy, segment $s_{i}^{u}$ is composed of $k$ segments $s_{j}^{u+1}(i)$ with respective fitting errors $E_{j}^{u+1}$. The composite error of the $k$ segments $s_{j}^{u+1}(i)$ at level $u$ is then defined as

$$
C E_{i}^{u}=\sum_{j} a_{j}^{u+1} E_{j}^{u+1} / \sum_{j} a_{j}^{u+1},
$$

where $a_{j}^{u+1}$ is the area (measured in amount of pixels) of segment $s_{j}^{u+1}$. We select $s_{i}^{u}$ at level $u$ if

$$
E_{i}^{u}<C E_{i}^{u}+\tau_{2}
$$

and the $k$ segments $s_{j}^{u+1}(i)$ from level $u+1$ otherwise. Parameter $\tau_{2}$ is introduced in order to avoid an oversegmentation of the image by preferring segments obtained at lower resolutions. The procedure is applied to each segment at level $u$. This way a new segmentation is constructed which replaces the initial segmentation at $u$ by a segmentation $u^{*}$.

Let us now consider a segmentation hierarchy consisting of $o$ levels, where $o$ is a parameter. We apply the selection procedure to the initial segmentations $u=o-1$ and $u=o$. The selection procedure is applied to the initial segmentation $u=o-2$ and $u^{*}=o-1$, and so on, until the end of the hierarchy is reached. In this paper, we choose a three-level hierarchy, i.e., $o=3$.

\section{Region merging}

Let us consider two segments $s_{i}$ and $s_{j}$ having fitting errors $E_{i}$ and $E_{j}$. Both segments are merged if $E_{i \cap j}<$ $\left(a_{i} E_{i}+a_{j} E_{j}\right) /\left(a_{i}+a_{j}\right)+\tau_{2}$, where $E_{i \cap j}$ is the fitting error of the merged segments and $a_{i}$ and $a_{j}$ is number of valid depth points in the area of the segments $s_{i}$ and $s_{j}$, respectively. This procedure allows merging of segments having different colors. The procedure is applied to all segments that are neighbors of each other (i.e. their closest pixels have to be less than $\tau_{3}=8$ pixels apart). When accepting a merge, segments are updated and the new segmentation is used when evaluating the remaining segment pairs.

\section{E. Region growing}

Let $p_{i}$ be a previously unclustered point with coordinates $\left(x_{i}, y_{i}, z_{i}\right)$ and color $\mathbf{c}_{i}$. We find all segment neighbors of this pixel within a radius of $\tau_{4}=5$ pixels. We compute the distance of $p_{i}$ to the surface of segment $s_{j}$ as $\operatorname{dist}_{i}^{j}=$ $\left|z_{i}-f_{j}\left(x_{i}, y_{i}\right)\right|$, where $f_{j}$ is the explicit surface-model function of segment $s_{j}$, and assign $p_{i}$ to the closest segment in the neighborhood. For points for which no depth value 
was originally measured we use the local mean depth value computed over a small area around the point.

\section{F. Fitting surface patches}

We choose two types of surfaces as surface models: planes and a quadratic function, which allows (among others) the modelling of spherical and cylindrical shapes. Surfaces with more involved curvatures could also be managed within the same approach, but are not required for the application at hand. Moreover, we use quadratic functions that allow computing depth $z$ explicitly for the $x-y$ coordinates in the form of $z=f(x, y)$.

1) Planes: Planar surfaces are described by three parameters $a, b$, and $c$, where the depth $z$ can be expressed as a function of $x$ and $y$ through

$$
z=a x+b y+c .
$$

2) Curved surfaces: Curved surfaces are described by five parameters $a, b, c, d$, and $e$, where the depth $z$ can be expressed as a function of $x$ and $y$ through

$$
z=a x^{2}+b y^{2}+c x+d y+e .
$$

\section{Plant MONitoring}

The segmentations together with the fitted surface models can be used to select points of interest (e.g., accessible leaves amenable to measuring or probing) and to move the camera to a suitable next viewpoint. Using the new viewpoint, the target area can be further examined and more information can be gathered.

\section{A. Locating candidate leaves}

For a given initial viewpoint, we select a set of candidate leaves by considering the size, color, and the fitting error of the extracted segments. Segments which are of sufficient green color and size are likely to represent a leaf. For this purpose, we use a simple thresholding procedure. If the mean green component is larger than both the mean red and mean blue component and larger than a value of 100 , and if the segment area contains more than 300 pixels, then the segment is considered to be a candidate leaf. The fitting error provides a confidence of this assertion. However, in the future we plan to use more sophisticated methods to select candidate leaves, e.g., by considering the shape of the segment. Leaf selection criteria may also be imposed through a given task. Selected segments which are in reach of the robot arm are exposed to further inspection by moving the robot towards closer to the target. This is done by finding the center point of the current target segment, and, using the fitted model, estimating the surface normal of this point. The 3D point together with the normal vector can then be used to calculate the desired new camera position.

\section{B. Computing a new viewpoint}

The goal position and orientation is in the RGB camera coordinate frame, and has to be expressed in the robot frame. Our approach uses the current robot position $\mathbf{H}_{\mathbf{t c p}}$ to compute the desired goal position $\mathbf{H}_{2}$ in the robot coordinate frame. The current 3D position of the leaf with respect to the camera system $\mathbf{H}_{11}$ is measured in the segmentation stage.

We have found that the best orientation for sensing is to place the camera system perpendicular to the leaf surface. Additionally, with this position we aim to minimize the difference in depth of the point cloud, as ToF cameras exhibit a depth error that depends on the current distance [10]. Moreover, we want the robot to avoid excessive rotations when changing point of view.

The desired global orientation of the camera $\mathbf{R}_{12}$ is computed using the cross product of the normal vector to the fitted surface in a selected point and the horizontal vector of the robot coordinate frame.

The translation vector $\mathbf{t}_{12}$ defines the desired position after the displacement, and depends on the task. It is defined as

$$
\mathbf{t}_{\mathbf{1 2}}=\left(g_{x} g_{y} g_{z}\right),
$$

where $g_{x}$ and $g_{y}$ are the displacement between the camera and the center of the tool that is used, and $g_{z}$ is the desired distance between the tool and the leaf at the end of the motion.

The desired transformation is then

$$
\mathbf{H}_{\mathbf{1 2}}=\left|\begin{array}{cc}
\mathbf{R}_{\mathbf{1 2}} & \mathbf{t}_{\mathbf{1 2}}{ }^{T} \\
0_{3} & 1
\end{array}\right| .
$$

The goal position $\mathbf{H}_{\mathbf{2}}$ can be found combining the previous transformations

$$
\mathbf{H}_{2}=\mathbf{H}_{\mathrm{tcp}} \mathbf{H}_{\mathrm{c}} \mathbf{H}_{11} \mathbf{H}_{12} \mathbf{H}_{\mathrm{c}}{ }^{-1},
$$

where $\mathbf{H}_{\mathbf{c}}$ is the transformation between the camera and the robot tool center point, that can be measured or calibrated with standard methods.

\section{EXPERIMENTAL RESULTS}

In the experiments, the robot has to examine a plant (placed on a table in the lab), select candidate leaves (using the algorithm described in Section IV), and move the camera system, mounted on the robot arm, to a new position, from which a better color/depth image of the target leaf can be obtained. Then, more information about the leaf is gathered.

The computations for segmenting the image and selecting target leaves are performed using MATLAB with an Intel Duo Core Processor T2250 of $1.73 \mathrm{GHz}$. Total run time of the algorithm using non-optimized code for segmenting the time-of-flight data is in the range of $\approx 1 \mathrm{~min}$ (including computation times for getting color segmentations). Parameters for segmenting time-of-flight data are $\tau_{1}=2 \mathrm{~cm}^{2}$ and $\tau_{2}=0.25 \mathrm{~cm}^{2}$.

We tested the method for different plants and viewpoints. In Fig. 4, the results of four experiments are collected. We explain the procedure using the first example shown in Fig. 4A. First, a color/depth image is acquired from the first (initial) viewing position of the robot. The color image is shown in the left upper panel of Fig. 4A. Next to it, the respective color-coded sparse depth data (presented in the image space of the RGB camera) is shown together with the computed segment boundaries (see upper middle panel). The 

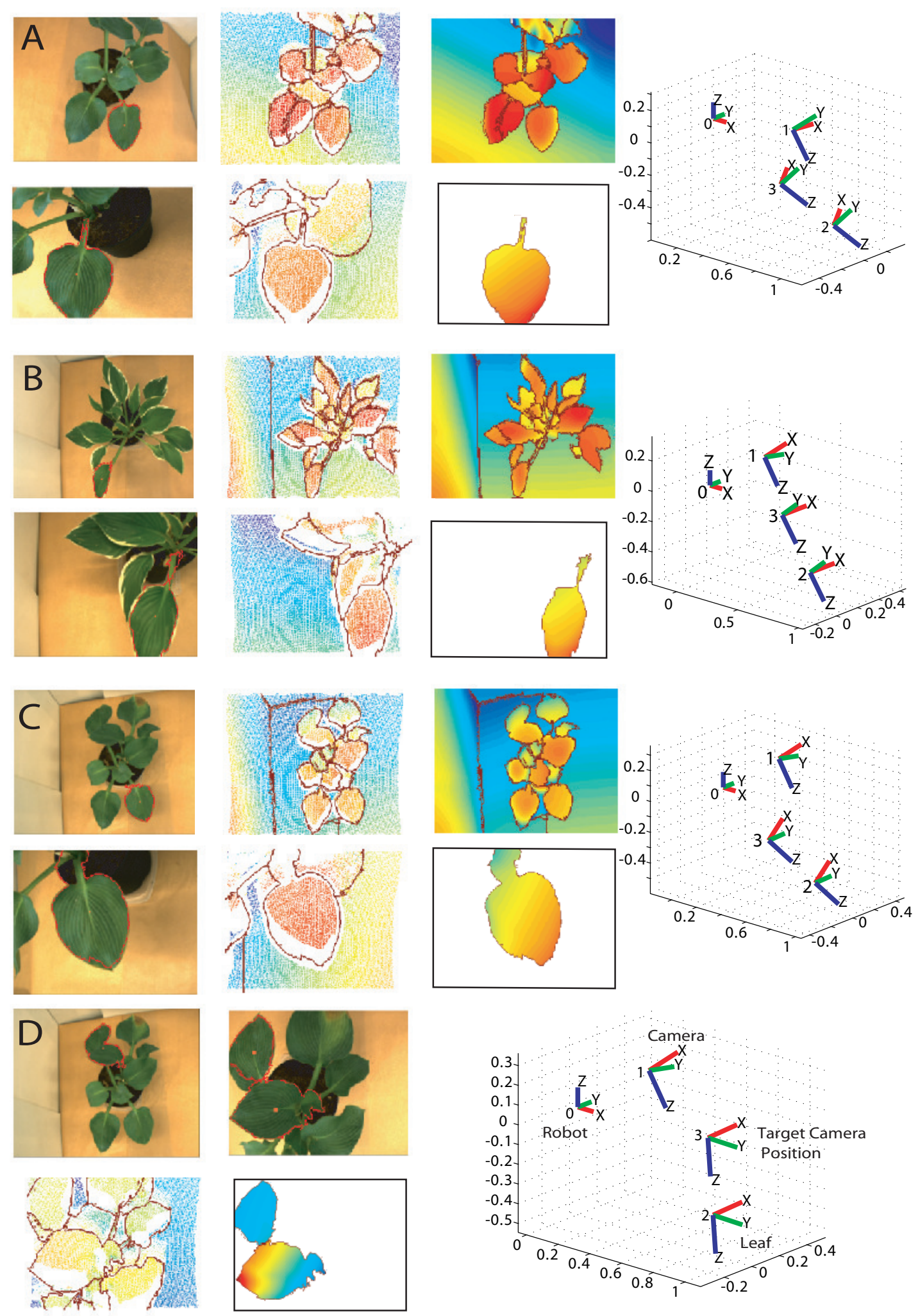

Fig. 4. Experimental results for different views of plants. A Color image first view (upper left panel). Sparse depth with segment boundaries (middle upper panel). Fitted depth (right upper panel). Color image close view (lower left panel). Sparse depth with segment boundaries of close view (middle lower panel). Fitted depth of selected segment (right lower panel). The selected segment is marked in red in the color images. A schematic showing the robot base position (0), the initial camera position (1), the leaf position (2) and the computed target position of the camera to capture the second viewpoint (3) are shown in the left panel. Distances are given in meters. Further examples are shown in B-D. 


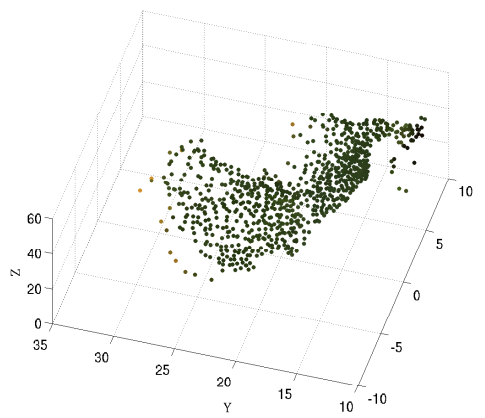

(A) Point cloud (first view)

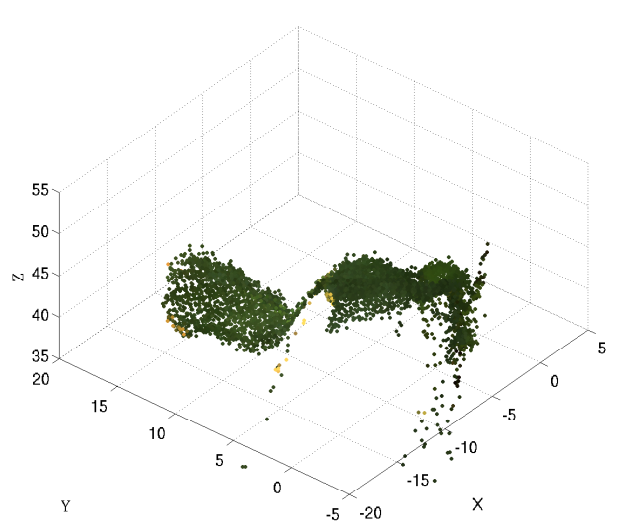

(C) Point cloud (close view)

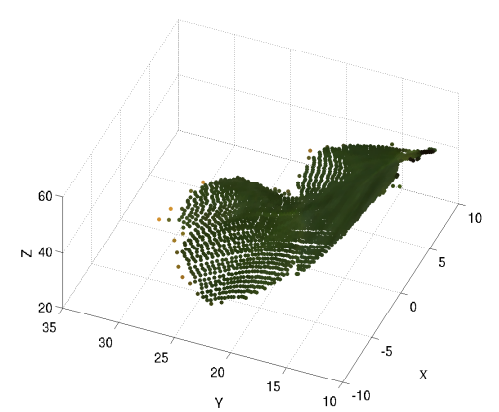

(B) Model point cloud (first view)

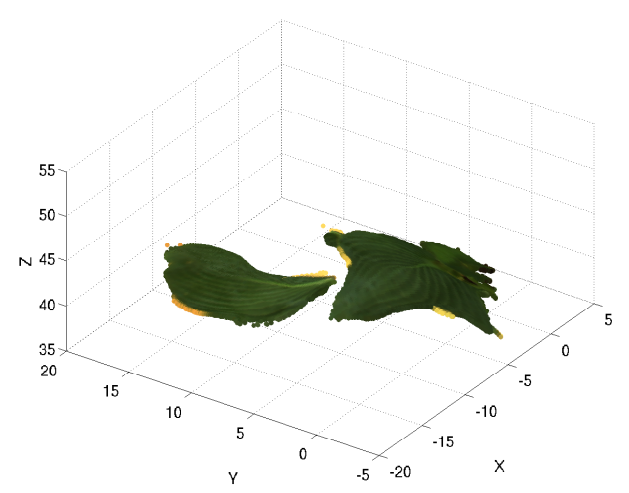

(D) Model point cloud (close view)

Fig. 5. Point clouds at different stages in the experiment of example D (see Fig 4). (A) Point cloud of a selected segment from the first camera viewpoint. (B) Model point cloud (after surface fitting) from the first camera viewpoint. (C) Point cloud from the second viewpoint. (D) Model point cloud (after surface fitting) from the second viewpoint. The distinct surfaces representing the two leaves can be distinguished. Distances here are given in centimeters.

fitted dense depth obtained via surface fitting is then shown in the right upper panel. The segmentation of the data into distinct surfaces provides us with an abstract representation which can be used to locate segments of interest. Such a segment is marked in red in the color image. We compute the surface normal of the segment and find the new target camera position, which is supposed to provide a better view on the selected segment. In the right panel of Fig. 4A, a schematic showing the robot base position (0), the initial camera position (1), the leaf position (2) and the computed target position of the camera (3) is shown. In the target camera position, the coordinate system of the camera is aligned with the coordinate system of the leaf.

After moving to the new camera position, a second color/depth image of the plant is obtained, which is shown in the lower left panel of Fig. 4A. The selected leaf is marked in red. The respective new segmentation is shown next to it in the lower middle panel. We also show the depth fitted to the leaf using the surface model in the lower right panel. We observe that a more detailed description of the target leaf is obtained by moving to the new camera position and applying our procedure. Similar results are obtained for the remaining examples shown in Fig. 4B-D.

We demonstrate that the method also allows to gather more semantic information about the plant. From the first view of the example shown in Fig. $4 \mathbf{C}$, a second candidate segment can be selected as shown in Fig. 4D, upper left panel. We see that two leaves are here merged into a single segment. By moving to the new viewpoint, we find that the original segment is composed of two segments, representing two distinct leaves, hence, our semantic knowledge about the plant could be refined. This is also illustrated in Fig. 5AD, where the colored point clouds for the first view, the second view, and after modeling the data of the second view of this example are shown, respectively. In the first view, the distinct surfaces of the leaves are hardly visible due to insufficient resolution. In the second view, however, the surfaces can be distinguished, and, using the segmentation procedure, separated, and fitted by a surface model.

Quantitative results in terms of segment magnification obtained in the second view, fitting errors, and surfaces types are summarized in Table I. Moving to a closer view allows to collect more data in the region of interest, and, as a consequence, better models can be fitted to the segments.

\section{CONCLUSIONS AND FUTURE WORK}

We presented a method for modeling and monitoring plant leaves using fused depth/color images acquired with 
TABLE I

SEgMENT MAGNIFICATION $M_{s}$ (COMPUTED AS THE RATIO OF THE SEGMENT SIZES IN THE SECOND AND FIRST VIEW), SQUARE FITTING ERRORS IN $\mathrm{CM}^{2}$, AND SURFACE TYPES FOR THE FIRST AND SECOND VIEWPOINT IN COMPARISON. FOR EXAMPLE D, THE RESULTS FOR THE TWO SEGMENTS OCCURING IN THE SECOND VIEW ARE SHOWN SEPARATELY.

\begin{tabular}{|c|c|c|c|c|c|}
\hline Example & $M_{s}$ & $E_{\text {view } 1}$ & $E_{\text {view 2 }}$ & Surface $_{\text {view 1 }}$ & Surface $_{\text {view 2 }}$ \\
\hline \hline $\mathrm{A}$ & 3.9 & 2.35 & 0.27 & Curved & Planar \\
\hline $\mathrm{B}$ & 6.4 & 3.72 & 1.09 & Curved & Planar \\
\hline $\mathrm{C}$ & 7.6 & 0.91 & 0.56 & Planar & Planar \\
\hline $\mathrm{D}$ & 6.3 & 4.27 & $1.21 / 2.43$ & Curved & Planar/Curved \\
\hline
\end{tabular}

a calibrated system of an RGB color camera and a PMD depth camera, based on the time-of-flight principle. Candidate leaves, selected using segment-based attributes such as size, color, and fitting error, are inspected by moving the robot arm, on which the camera system is mounted, to a new position, which allows obtaining a better view of the segment. This way, more information about the leaf is gathered. For example, two leaves that have been initially merged into a single segment due to insufficient resolution available in the first view, could be separated and modeled from the second view, extending the semantic knowledge of this area of the plant (see Fig. 5).

In the described approach, planar and curved quadric surface models are used to guide the segmentation process, which is conducted in the color space. This way, also sparse or noisy data can be used. This kind of data often poses a problem to approaches working in the depth space directly. More domain specific models, e.g., as described in [16], could in principle be included in the procedure. But, this would have to be done with some care, since segments appearing at intermediate stages of the process often only represent leaf fragments.

The method relies on fused depth/color images which are brought in correspondence despite their slightly different positions and viewing angles, giving rise to occlusions, which have to be detected accurately for the method to work properly. In particular for close views, this problem can be severe and caused the method to fail in a few cases. We have observed that as we get close to the plant some leaves can move out of the viewing zone of one of the cameras. The relative orientation between the ToF and the RGB cameras can be reconsidered to gain a better overlap between both images.

Fusing depth with color provides not only additional important information about the plant, but also allows us to perform the segmentation in the dense color space, and, doing this, to cope with characteristic properties of the ToF data, i.e. low resolution. In the future, we intend to replace the color segmentation algorithm by a real-time algorithm to improve processing times. To the authors' knowledge, this is the first time that an active vision approach, using ToF depth and color, has been applied to robotized plant measuring. In the future, we aim to take probes from leaves selected by our method. From the close viewpoint, a probing tool can be guided to the surface of the leaf and samples can be taken.

We have experienced some problems due to the different point of view of each camera, and principally due to their different viewing zone, that leads to a reduced common sensing area. We are now considering to change the distribution of the camera system to maximize the common sensing area mounting the cameras at $90^{\circ}$ and using a beam splitter to obtain common image centers and viewing directions.

\section{REFERENCES}

[1] A. Kolb, E. Barth, and R. Koch, "Tof-sensors: New dimensions for realism and interactivity," in IEEE CVPRW, 2008, pp. 1518-1523.

[2] A. Nealen, M. Muller, R. Keiser, E. Boxerman, and M. Carlson, "Physically based deformable models in computer graphics," Computer Graphics Forum, vol. 25, no. 4, pp. 809-816, 2006.

[3] R. Rusu, A. Holzbach, R. Diankov, G. Bradski, and M. Beetz, "Perception for mobile manipulation and grasping using active stereo," in 9th IEEE-RAS Intl. Conf. on Humanoid Robots, 2009, pp. 632-638.

[4] T. Fourcaud, X. Zhang, A. Stokes, H. Lambers, and C. Köner, "Plant growth modelling and applications: The increasing importance of plant architecture in growth models," Annals of Botany, vol. 101, pp. 10531063, 2008.

[5] L. Quan, P. Tan, G. Zeng, L. Yuan, J. Wang, and S. Kang, "Imagebased plant modelling," in ACM Siggraph, 2006, pp. 599-604.

[6] Y. Song, R. Wilson, R. Edmondson, and N. Parsons, "Surface modelling of plants from stereo images," in 6th IEEE Intl. Conf. on $3 D$ Digital Imaging and Modelling, 2007.

[7] G. Taylor and L. Kleeman, "Robust range data segmentation using geometric primitives for robotic applications," in 5th Iasted Int. Conf. on Signal and Image Processing, 2003.

[8] R. Klose, J. Penlington, and A. Ruckelshausen, "Usability study of 3d time-of-flight cameras for automatic plant phenotyping," in Workshop on Computer Image Analysis in Agriculture, 2009, pp. 93-105.

[9] S. Foix, G. Alenya, and C. Torras, "Lock-in time-of-flight (tof) cameras: A survey," Sensors Journal, IEEE, 2011

[10] S. Foix, G. Alenyà, J. Andrade-Cetto, and C. Torras, "Object modeling using a tof camera under an uncertainty reduction approach," in IEEE Intl. Conf. on Robotics and Automation, 2010, pp. 1306-1312.

[11] B. Bartczak and R. Koch, "Dense depth maps from low resolution time-of-flight depth and high resolution color views," ser. LNCS, no. 5876, 2009, pp. 228-239.

[12] A. Bleiweiss and M. Werman, "Fusing time-of-flight depth and color for real-time segmentation and tracking," in Workshop on Dynamic 3D Imaging (Dyn3D'09), 2009, pp. 58-69.

[13] F. Khalil and P. Payeur, Robot Manipulators Trends and Development INTECH, 2010, ch. Dexterous Robotic Manipulation of Deformable Objects with Multi-Sensory Feedback - a Review, pp. 587-619.

[14] B. Bartczak, I. Schiller, C. Beder, and R. Koch, "Integration of a timeof-flight camera into a mixed reality system for handling dynamic scenes, moving viewpoints and occlusions in real-time," in Int. Sym. 3D Data Processing, Visualization and Transmission (3DPVT), 2008

[15] B. Dellen and F. Woergoetter, "Disparity from stereo-segment silhouettes of weakly-textured images," in BMVC, 2009.

[16] T. B. Moeslund, M. Aagaard, and D. Lerche, " $3 \mathrm{~d}$ pose estimation of cactus leaves using an active shape model," in Proceedings of the Seventh IEEE Workshop on Applications of Computer Vision, 2005. 